\title{
Scanner Identification Using Sensor Pattern Noise
}

\author{
Nitin Khanna ${ }^{a}$, Aravind K. Mikkilineni ${ }^{b}$ \\ George T. C. Chiu ${ }^{b}$, Jan P. Allebach ${ }^{a}$, Edward J. Delp ${ }^{a}$ \\ ${ }^{a}$ School of Electrical and Computer Engineering \\ ${ }^{b}$ School of Mechanical Engineering \\ Purdue University, West Lafayette, Indiana USA
}

\begin{abstract}
Digital images can be captured or generated by a variety of sources including digital cameras and scanners. In many cases it is important to be able to determine the source of a digital image. This paper presents methods for authenticating images that have been acquired using flatbed desktop scanners. The method is based on using the pattern noise of the imaging sensor as a fingerprint for the scanner, similar to methods that have been reported for identifying digital cameras. To identify the source scanner of an image a reference pattern is estimated for each scanner and is treated as a unique fingerprint of the scanner. An anisotropic local polynomial estimator is used for obtaining the reference patterns. To further improve the classification accuracy a feature vector based approach using an SVM classifier is used to classify the pattern noise. This feature vector based approach is shown to achieve a high classification accuracy.
\end{abstract}

Keywords: digital forensics, imaging sensor classification, flatbed scanner, sensor noise, scanner forensics

\section{INTRODUCTION}

Advances in digital imaging technologies have led to the development of low-cost and high-resolution digital cameras and scanners. Both digital cameras and desktop scanners are becoming ubiquitous. Digital images produced by various sources are widely used in a number of applications from medical imaging and law enforcement to banking and daily consumer use. The increasing functionality of image editing software allows even a beginner to easily manipulate images. In some cases a digitally scanned image can meet the threshold definition requirements of a "legal duplicate" if the document can be properly authenticated [1]. Forensic tools that help establish the origin, authenticity, and the chain of custody of digital images are essential to a forensic examiner. These tools can prove to be vital whenever questions of digital image integrity are raised. Therefore, a reliable and objective way to examine digital image authenticity is needed.

There are various levels at which the image source identification problem can be addressed. One may want to find the particular device (digital camera or scanner) which generated the image or one might be interested in knowing only the make and model of the device (digital camera or scanner). As summarized in [2], a number of very interesting and robust methods have been proposed for source camera identification [3-6]. In [7], a novel technique for classification of images based on their sources, scanned and non-scanned images, is presented.

To the best of our knowledge, no prior work presents methods specifically for scanner identification. In this paper we will extend the methods for source camera identification to scanners. One approach for digital camera identification is based on characterizing the imaging sensor used in the device. In [8], it is shown that defective pixels can be used for reliable camera identification even from lossy compressed images. This type of noise, generated by hot or dead pixels, is typically more prevalent in cheap cameras. The noise can be visualized by averaging multiple images from the same camera. These errors can remain visible after the image is compressed. Many cameras post-process the captured image to remove these types of noise, so this technique cannot always be used.

In [6], an approach for camera identification using the imaging sensor's pattern noise was presented. The identification is based on pixel nonuniformity noise which is a unique stochastic characteristic for both CCD

This research was supported by a grant from the National Science Foundation, under Award Number 0524540. Address all correspondence to E. J. Delp at ace@ecn.purdue.edu

Security, Steganography, and Watermarking of Multimedia Contents IX, edited by Edward J. Delp III, Ping Wah Wong, Proc. of SPIE-IS\&T Electronic Imaging, SPIE Vol. 6505, 65051K, @ 2007 SPIE-IS\&T · 0277-786X/07/\$18 
(Charged Coupled Device) and CMOS (Complementary Metal Oxide Semiconductor) sensors. Reliable identification is possible even from images that are resampled and JPEG compressed. The pattern noise is caused by several factors such as pixel non-uniformity, dust specks on the optics, optical interference, and dark current [9]. The high frequency part of the pattern noise is estimated by subtracting a denoised version of the image from the original. This is performed using a wavelet based denoising filter [10]. A camera's reference pattern is determined by averaging the noise patterns from multiple images obtained from the camera. This reference pattern serves as an intrinsic signature of the camera. To identify the source camera, the noise pattern from an image is correlated with known reference patterns from a set of cameras and the camera corresponding to the reference pattern giving maximum correlation is chosen to be the source camera.

In this paper we will present methods for authenticating images that have been captured by flatbed desktop scanners using sensor pattern noise. Initially, a correlation based approach for authenticating digital cameras [6] is extended for source scanner identification. We will also describe the use of a SVM classifier to classify the images based on feature vectors obtained from the sensor pattern noise. As shown by the experimental results, this feature vector based approach gives much better classification accuracy than correlation based approaches.

\section{SCANNER OVERVIEW}

\subsection{Scanner Imaging Pipeline}

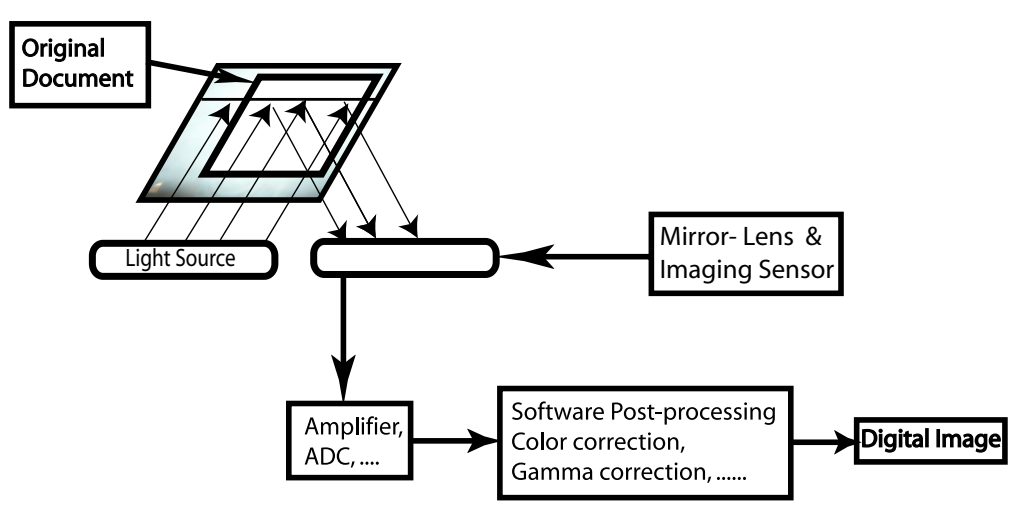

Figure 1. Flatbed Scanner Imaging Pipeline

Figure 1 shows the basic structure of a flatbed scanner's imaging pipeline[11,12]. The document is placed in the scanner and the acquisition process starts. The lamp used to illuminate the document is either a cold cathode fluorescent lamp (CCFL) or a xenon lamp, older scanners may have a standard fluorescent lamp. Using a stabilizer bar, a belt, and a stepper motor, the scan head slowly translates linearly to capture the image. The purpose of the stabilizer bar is to ensure that there is no wobble or deviation in the scan head with respect to the document. The scan head includes a set of lenses, mirrors, a set of filters, and the imaging sensor. Most desktop scanners use charge-coupled device (CCD) imaging sensors. Other scanners use CMOS (complementary metal oxide semiconductor) imaging sensors, Contact Image Sensors (CIS), or PMTs (photomultiplier tube) [11, 12]. The maximum resolution of the scanner is determined by the horizontal and vertical resolution. The number of elements in the linear CCD sensor determines the horizontal optical resolution. The step size of the motor controlling the scan head dictates the vertical resolution.

There are two basic methods for scanning an image at a resolution lower than the hardware resolution of the scanner. One approach is to sub-sample the output of the sensor. Another approach involves scanning at the full resolution of the sensor and then down-sampling the results in the scanner's memory. Most good quality scanners adopt the second method since it yields far more accurate results. 


\subsection{Sensor Noise}

The manufacturing process of imaging sensors introduces various defects which create noise in the pixel values $[9,13]$. There are two types of noise which are important. The first type of noise is caused by array defects. These include point defects, hot point defects, dead pixels, pixel traps, column defects and cluster defects. These defects cause pixel values in the image to deviate greatly. For example, dead pixels show up as black in the image and hot point defects show up as very bright pixels in the image, regardless of image content. Pattern noise refers to any spatial pattern that does not change significantly from image to image and is caused by dark currents and photoresponse nonuniformity (PRNU). Dark currents are stray currents from the sensor substrate into the individual pixels. This varies from pixel to pixel and the variation is known as fixed pattern noise (FPN). FPN is due to differences in detector size, doping density, and foreign matter trapped during fabrication. PRNU is the variation in pixel responsivity and is seen when the device is illuminated. This noise is due to variations between pixels such as detector size, spectral response, thickness in coatings and other imperfections created during the manufacturing process. Frame averaging will reduce the noise sources except for FPN and PRNU. Although FPN and PRNU are different, they are collectively known as scene noise, pixel noise, pixel nonuniformity, or simply pattern noise.

Both digital cameras and scanners work on similar basic principles in terms of the imaging sensor. However there is an important difference in the geometry of the sensor used by the two devices. Digital cameras use a two dimensional sensor array while most of the present day scanners use a linear sensor array. In the case of flatbed scanners, the same linear array is translated to generate the entire image. Furthermore, for a digital camera all the sensor elements are used simultaneously, while for scanning only a portion of the linear sensor generates the complete image.

The fixed component of the sensor noise can be used for source scanner identification. In [14] a method of estimating sensor noise is successfully used for source camera identification. This proposed method used a wavelet filter in combination with frame averaging to estimate the pattern noise in an image. This method is the basis for our scanner identification techniques.

\section{CORRELATION BASED APPROACHES}

Both digital cameras and many flatbed scanners use CCD imaging sensors. The imaging sensor's pattern noise has been successfully used for source camera identification as described in [6]. The sensor noise as described in the previous sections can be modeled as the sum of two components; a random component and a fixed component. By random component we refer to that portion of noise which changes from image to image and varies over a period of time, while the fixed component of noise is a characteristic of the imaging sensor and it remains the same from image to image. The challenge is to separate the random component from the fixed component of the noise. The high frequency part of the pattern noise is estimated by subtracting a denoised version of an image from the original image [6].

For denoising we used an anisotropic local polynomial estimator based on directional multiscale optimizations[15]. Experiments were performed with two types of denoising filters, a wavelet based denoising filter [10] as reported in [6] and the polynomial estimator [15]. Initial experiments indicate that the polynomial estimator may be more robust for use in scanners. A scanner's reference pattern is determined by averaging the noise patterns from multiple images captured by the scanner. This reference pattern serves as an intrinsic signature of the scanner (Figure 2). To identify the source scanner, the noise pattern from an image is correlated with known reference patterns from a set of scanners (Figure 3). The scanner corresponding to the reference pattern with highest correlation is chosen to be the source scanner[6].

In contrast to digital cameras, flatbed scanners use a linear one dimensional sensor array. Using a one dimensional version of the two dimensional array reference pattern described in $[6]$ is more appropriate in this case. The linear sensor noise pattern is obtained from the average of all the rows of the noise corresponding to the image. Finally, the linear sensor reference noise pattern for a particular scanner is obtained by taking the average of linear sensor noise patterns of multiple images scanned by the same scanner. This linear row reference pattern serves as an intrinsic signature of the scanner. To identify the source scanner, the linear noise pattern 


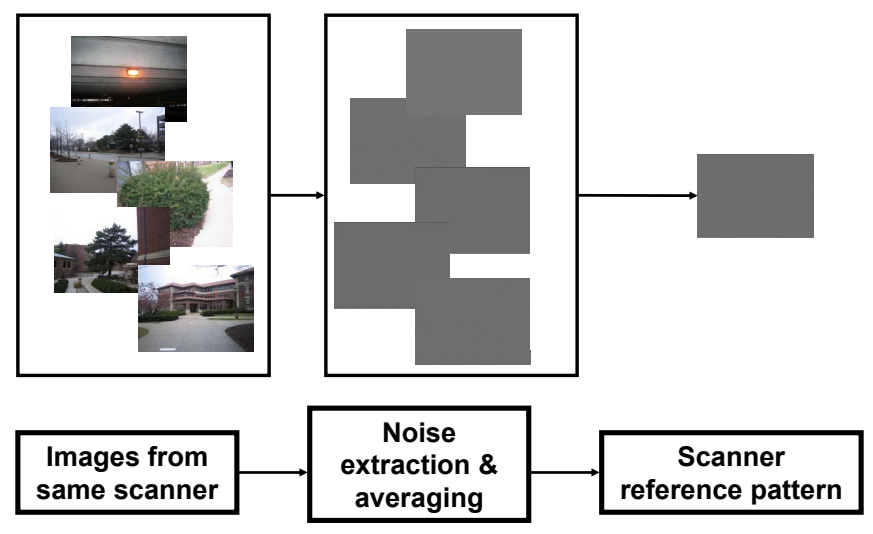

Figure 2. Classifier Training for Correlation-Based Approach.
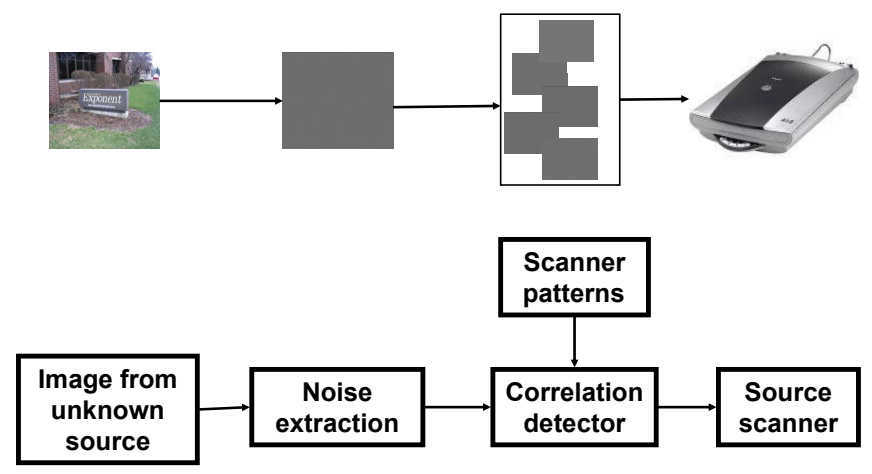

Figure 3. Source Scanner Identification Using A Correlation-Based Detection Scheme.

from an image is correlated with known reference patterns from a set of scanners[6]. The scanner corresponding to the reference pattern with highest correlation is chosen to be the source scanner.

Let $I^{k}$ denote the $k^{t h}$ input image of size $M * N$ pixels ( $M$ rows and $N$ columns). Let $I_{\text {noise }}^{k}$ be the noise corresponding to the original input image $I^{k}$ and let $I_{\text {denoised }}^{k}$ be the result of using the denoising filter on $I$. Then as in [14],

$$
I_{\text {noise }}^{k}=I^{k}-I_{\text {denoised }}^{k}
$$

Let $K$ be the number of images used to obtain the reference pattern of a particular scanner. Then the two dimensional array reference pattern is obtained as

$$
\widetilde{I}_{\text {noise }}^{\text {array }}(i, j)=\frac{\sum_{k=1}^{K} I_{\text {noise }}^{k}(i, j)}{K} ; 1 \leq i \leq M, 1 \leq j \leq N
$$

The linear row reference pattern is obtained as

$$
\widetilde{I}_{\text {noise }}^{\text {inear }}(1, j)=\frac{\sum_{k=1}^{K} \sum_{i=1}^{M} I_{\text {noise }}^{k}(i, j)}{M * K} ; 1 \leq j \leq N
$$

Correlation is used as a measure of the similarity between the scanner reference patterns and the noise pattern of a given image[6]. Correlation between two vectors $\mathbf{X}, \mathbf{Y} \in \mathbb{R}^{N}$ is defined as 


$$
\operatorname{correlation}(X, Y)=\frac{(X-\bar{X}) \cdot(Y-\bar{Y})}{\|X-\bar{X}\| \cdot\|Y-\bar{Y}\|}
$$

This correlation is used to classify the scanners.

\section{THE USE OF STATISTICAL FEATURES OF THE PATTERN NOISE}

Due to the following reasons we believe correlation based approaches are not the best method for scanner identification:

- Using correlation as a measure of similarity is highly sensitive to de-synchronization, which is almost unavoidable in the case of flatbed scanners since it is very difficult to place a document at the exact same location on the scanner bed twice.

- Most documents to be scanned cover only a part of the scanner bed so not all the CCD elements are involved in scanning.

Instead of using correlation as a measure of similarity, statistical features can be extracted from the pattern noise and used for classification by a Support Vector Machine (SVM).

\subsection{Feature Vector Selection}

For scanned images, the average row will give an estimate of the fixed "row-pattern" of the sensor noise since averaging will reduce the random component while at the same time enhancing the fixed component of the noise. To address the two points in the previous section, we propose to use two sets of eight features extracted from the sensor pattern noise.

Statistical properties of the average row such as mean, median, standard deviation, skewness, and kurtosis are used as the first set of features. It is expected that there is a periodicity between different rows of the fixed component of the sensor noise of a scanned image. To detect the similarity between different rows of the noise, the correlation between each of the $M$ rows of the sensor noise with the average row are obtained. The second set of features is obtained from the statistical properties of these correlations. In total these features form a sixteen dimensional feature vector for each scanned image. These features capture the essential properties of the image which are useful for discriminating between two scanners. The second set of features represent the proportion of the fixed component of the pattern noise. For a low quality scanner, having a large amount of random noise such as that due to fluctuations in lighting conditions, the inter-row correlations will be quite small as compared to a very high quality scanner.

\subsection{Support Vector Machine}

Suppose we are given training data $\left(\mathbf{x}_{1}, y_{1}\right), \ldots,\left(\mathbf{x}_{n}, y_{n}\right)$ where $y_{i} \in\{1,-1\}$. The vectors $\mathbf{x}_{i}, \forall i$ represent the feature vectors input to the SVM classifier and $y_{i}$ represent the corresponding class labels. Assuming that the class represented by the subset $y_{i}=1$ and the class represented by $y_{i}=-1$ are "linearly separable", the equation of a decision surface in the form of a hyperplane that does the separation is $\mathbf{w}^{T} \mathbf{x}+b=0$; where, $\mathbf{x}$ is an input vector, $\mathbf{w}$ is an adjustable weight vector, and $b$ is a bias.

For a given weight vector $\mathbf{w}$ and bias $b$, the separation between the hyperplane and the closest data point is known as the margin of separation, denoted by $M$. The goal of a support vector machine is to find the particular hyperplane for which the margin of separation $M$ is maximized[16]. Under this condition the decision surface is referred to as the optimum separating hyperplane $(\mathrm{OSH})\left(\mathbf{w}_{o}^{T} \mathbf{x}+b_{o}=0\right)$.

The pair $\left(\mathbf{w}_{o}, b_{o}\right)$ with appropriate scaling, must satisfy the constraint:

$$
\begin{gathered}
\mathbf{w}_{o}^{T} \mathbf{x}+b_{o} \geq 1 \quad \forall y_{i}=+1 \\
\mathbf{w}_{o}^{T} \mathbf{x}+b_{o} \leq-1 \quad \forall y_{i}=-1
\end{gathered}
$$


The particular data points $\left(\mathbf{x}_{i}, y_{i}\right)$ for which $y_{i}\left[\mathbf{w}^{T} \mathbf{x}_{i}+b\right]=1$ are known as support vectors, hence the name "Support Vector Machine." The support vectors are the data points that lie closest to the decision surface and are therefore the most difficult to classify. As such they have the direct bearing on the optimum location of the decision surface. Since the distance to the closest point is $\frac{1}{\|\mathbf{w}\|}$, finding the OSH amounts to minimizing $\|\mathbf{w}\|$ with the objective function: $\min \phi(\mathbf{w})=\frac{1}{2}\|\mathbf{w}\|^{2}$ subject to the constraints shown in Equations 5 and 6 .

If $\left(\alpha_{1}, \alpha_{2} \ldots, \alpha_{N}\right)$ are the $N$ non-negative Lagrange multipliers associated with constraints in Equations 5 and 6 , the OSH can be uniquely constructed by solving a constrained quadratic programming problem. The solution $\mathbf{w}$ has an expansion $\mathbf{w}=\sum_{i} \alpha_{i} y_{i} \mathbf{x}_{i}$ in terms of a subset of training patterns, known as support vectors, which lie on the margin. The classification function can thus be written as

$$
f(\mathbf{x})=\operatorname{sgn}\left(\sum_{i} \alpha_{i} y_{i} \mathbf{x}_{i}^{T} \mathbf{x}+b\right)
$$

If the data is not linearly separable, SVM introduces slack variables and a penalty factor such that the objective function can be modified as

$$
\phi(\mathbf{w})=\frac{1}{2}\|\mathbf{w}\|^{2}+C\left(\sum_{i=1}^{N} \zeta_{i}\right)
$$

Additionally, the input data can be mapped through some nonlinear mapping into a higher-dimensional feature space in which the optimal separating hyperplane is constructed. Thus the dot product required in Equation 7 can be represented by $k(\mathbf{x}, \mathbf{y})=(\phi(\mathbf{x}) . \phi(\mathbf{y}))$ when the kernel $k$ satisfies Mercer's condition[17]. Finally, the classification function is obtained as

$$
f(\mathbf{x})=\operatorname{sgn}\left(\sum_{i} \alpha_{i} \mathbf{y}_{i} k\left(\mathbf{x}_{i}, \mathbf{x}\right)+b\right)
$$

In the above, the classification of input vectors into two classes was described. The generalization to $m$-class classifier is discussed below. Recall that the SVM uses OSHs to divide the input vectors into two classes. One of the solutions to the multi-class classification problem is to train $m$ SVMs, each responsible for separating the $j^{\text {th }}$ class from the rest.

Suppose the training data is $\left(\mathbf{x}_{1}, y_{1}\right), \ldots,\left(\mathbf{x}_{n}, y_{n}\right)$ where $y_{i} \in\{1,2, \ldots, m\}$. Let us define $y_{i}^{j},($ for $j=1,2, \ldots, m$ and $i=1,2, \ldots, n)$

$$
y_{i}^{j}= \begin{cases}1, & \text { if } y_{i}=j ; \\ -1, & \text { otherwise }\end{cases}
$$

The SVM training algorithm learns the function $f^{j}$ which corresponds to the $j^{\text {th }}$ SVM according to the following equation

$$
f^{j}(\mathbf{x})=\operatorname{sgn}\left(\sum_{i} \alpha_{i}^{j} y_{i}^{j} k^{j}\left(\mathbf{x}_{i}, \mathbf{x}\right)+b^{j}\right) \quad j=1,2, \ldots, m
$$

Note that each of these SVMs could potentially use different kernels, $k^{j}$, and bias $b^{j}$.

Given an input vector $\mathbf{x}$, it is classified as

$$
y=\underbrace{\operatorname{argmax}}_{j} f^{j}(\mathbf{x})
$$

Because the SVM can be analyzed theoretically using concepts from statistical learning theory, it has particular advantage in problems with limited training samples in high-dimensional space. 


\section{EXPERIMENTAL RESULTS}

Table 1 shows the scanners used in our experiments. Approximately 25 images are scanned with each of the 4 scanners (a total of approximately 100 images) at the native resolution of the scanners. These images are then sliced into blocks of size $1024 \times 768$ pixels. Thus, in total, we have approximately 1200 scanned sub-images. Figure 5 shows a sample of the images used in this study. As shown in Figure 4 the image blocks such as $B 0$ and $B 5$ from the same columns will be scanned by the exact same sensor elements.

Table 1. Image Sources Used in Experiments

\begin{tabular}{|c|c|c|c|c|c|}
\hline & Device Brand & Type & Sensor & Native Resolution & Image Format \\
\hline \hline$S_{1}$ & Epson Perfection 4490 Photo & Flatbed Scanner & CCD & $4800 \mathrm{dpi}$ & TIFF \\
\hline$S_{2}$ & HP ScanJet $6300 \mathrm{c}-1$ & Flatbed Scanner & CCD & $1200 \mathrm{dpi}$ & TIFF \\
\hline$S_{3}$ & HP ScanJet $6300 \mathrm{c}-2$ & Flatbed Scanner & CCD & $1200 \mathrm{dpi}$ & TIFF \\
\hline$S_{4}$ & HP ScanJet 8250 & Flatbed Scanner & CCD & $4800 \mathrm{dpi}$ & TIFF \\
\hline
\end{tabular}

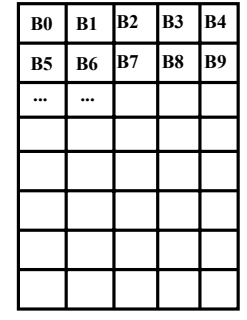

Figure 4. Scanned Images Are Sliced Into Sub-Images.
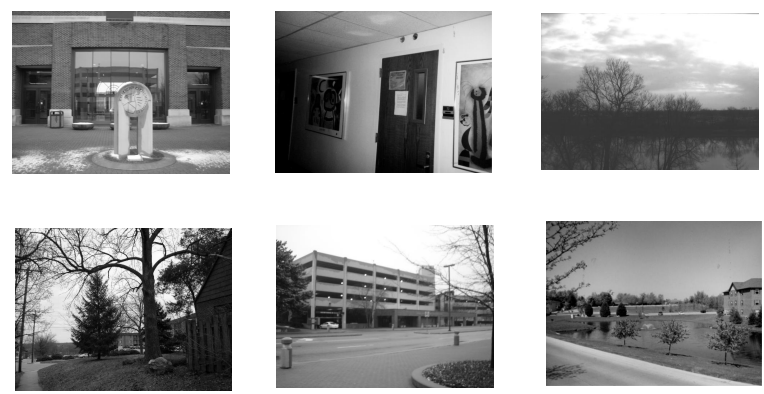

Figure 5. Sample Images.

\subsection{Experiment 1 : 2-D Reference Pattern}

In this experiment approximately 300 sub-images from each of the four scanners are used. One hundred randomly chosen sub-images (from each scanner) are used to estimate the two dimensional array reference patterns. Testing is performed using the remaining images. The anisotropic local polynomial estimator based denoising method (LPA-ICI) [15] is used to extract the noise from the images and the source scanner is determined using correlation between the estimated 2-D noise and reference patterns.

Tables 2 and 3 show the confusion matrix for the classification between pairs of scanner models. The $(i, j)^{t h}$ entry of the confusion matrix denotes the percentage of images which belong to the $i^{\text {th }}$ class but are classified as coming from the $j^{t h}$ class. Using the two dimensional array reference pattern gives an average classification accuracy of $72 \%$ and $84.5 \%$, for the scanner pairs $\left(S_{1}, S_{2}\right)$ and $\left(S_{2}, S_{4}\right)$ respectively. For separtion between two scanners these results are not very encouraging since for practical scenarios classification among large number of scanners is needed.

Table 2. Confusion Matrix for Experiment 1 (2D Reference Pattern)

\begin{tabular}{|c|c|c|c|}
\hline \multicolumn{2}{|c|}{} & \multicolumn{2}{c|}{ Predicted } \\
\cline { 3 - 4 } \multicolumn{2}{|c|}{} & $S_{1}$ & $S_{2}$ \\
\hline \hline \multirow{2}{*}{ Actual } & $S_{1}$ & 66.8 & 33.2 \\
\cline { 2 - 4 } & $S_{2}$ & 22.5 & 77.5 \\
\hline
\end{tabular}

Table 3. Confusion Matrix for Experiment 1 (2D Reference Pattern)

\begin{tabular}{|c|c|c|c|}
\hline \multicolumn{2}{|c|}{} & \multicolumn{2}{c|}{ Predicted } \\
\cline { 3 - 4 } \multicolumn{2}{|c|}{} & $S_{2}$ & $S_{4}$ \\
\hline \hline \multirow{2}{*}{ Actual } & $S_{2}$ & 69.4 & 30.6 \\
\cline { 2 - 4 } & $S_{4}$ & 0.4 & 99.6 \\
\hline
\end{tabular}


Table 4. Confusion Matrix for Experiment 2 (1D Reference Pattern)

\begin{tabular}{|c|c|c|c|}
\hline \multicolumn{2}{|c|}{} & \multicolumn{2}{c|}{ Predicted } \\
\cline { 3 - 4 } \multicolumn{2}{|c|}{} & $S_{1}$ & $S_{2}$ \\
\hline \hline \multirow{2}{*}{ Actual } & $S_{1}$ & 63.7 & 36.3 \\
\cline { 2 - 4 } & $S_{2}$ & 21.6 & 78.4 \\
\hline
\end{tabular}

Table 6. Confusion Matrix for Experiment 1 (2D Reference Pattern)

\begin{tabular}{|c|c|c|c|c|}
\hline \multicolumn{2}{|c|}{} & \multicolumn{3}{|c|}{ Predicted } \\
\cline { 3 - 5 } \multicolumn{2}{c|}{} & $S_{1}$ & $S_{2}$ & $S_{4}$ \\
\hline \hline \multirow{3}{*}{ Actual } & $S_{1}$ & 63.3 & 20.8 & 15.8 \\
\cline { 2 - 5 } & $S_{2}$ & 11.7 & 59.5 & 28.8 \\
\cline { 2 - 5 } & $S_{4}$ & 0.4 & 0.0 & 99.6 \\
\hline
\end{tabular}

Table 5. Confusion Matrix for Experiment 2 (1D Reference Pattern)

\begin{tabular}{|c|c|c|c|}
\hline \multicolumn{2}{|c|}{} & \multicolumn{2}{c|}{ Predicted } \\
\cline { 3 - 4 } \multicolumn{2}{c|}{} & $S_{2}$ & $S_{4}$ \\
\hline \hline \multirow{2}{*}{ Actual } & $S_{2}$ & 85.1 & 14.9 \\
\cline { 2 - 4 } & $S_{4}$ & 0.0 & 100.0 \\
\hline
\end{tabular}

Table 7. Confusion Matrix for Experiment 2 (1D Reference Pattern)

\begin{tabular}{|c|c|c|c|c|}
\hline \multicolumn{2}{|c|}{} & \multicolumn{3}{|c|}{ Predicted } \\
\cline { 3 - 5 } \multicolumn{2}{|c|}{} & $S_{1}$ & $S_{2}$ & $S_{4}$ \\
\hline \hline \multirow{3}{*}{ Actual } & $S_{1}$ & 63.3 & 23.6 & 13.1 \\
\cline { 2 - 5 } & $S_{2}$ & 10.8 & 69.4 & 19.8 \\
\cline { 2 - 5 } & $S_{4}$ & 0.0 & 0.0 & 100.0 \\
\hline
\end{tabular}

\subsection{Experiment 2 : 1-D Reference Pattern}

The same images as those used for Experiment 1 are used in this experiment. One hundred randomly chosen sub-images (from each scanner) are used for estimation of the one dimensional row reference patterns. The source class in this case is determined through correlation of the 1-D noise and reference patterns. Tables 4 and 5 show the confusion matrix for classification between pairs of scanner models. Using the one dimensional row reference pattern gives an average classification accuracy of $71 \%$ and $92.5 \%$, for the scanner pairs $\left(S_{1}, S_{2}\right)$ and $\left(S_{2}, S_{4}\right)$ respectively.

Tables 6 and 7 show the confusion matrices for source scanner identification among three scanners by using the two dimensional array reference patterns and one dimensional row reference patterns respectively. For classification among these three scanners, using the array reference pattern gives an average classification accuracy of $74 \%$ while using the row reference pattern gives an average classification acuracy of $77.6 \%$.

As discussed in Section 3, the results presented in this section imply that using the row reference pattern gives better results for source scanner identification than the two dimensional array reference pattern.

\subsection{Experiment 3 : SVM}

The experimental procedure for the feature based classification approach is shown in Figure 6 . The $S V M^{l i g h t}$ package [18] is used in this study. A radial basis function is chosen as the kernel function.

Approximately 300 sub-images from each of the four scanners is used. From these, $50 \%$ are used for training and rest for testing the SVM classifier. A total of 16 features are extracted from each of the images.

Table 8 shows the confusion matrix for classifying the sub-images coming from two columns of the same scanner. Possible reasons for poor classification accuracy are that images of both the classes are affected by similar mechanical fluctuations and are generated using the exact same post-processing algorithms.

Table 9 shows the confusion matrix for classifying images coming from the first columns of two different scanners. The average classification accuracy in this case is $99.5 \%$. Table 10 and Table 11 show the confusion matrices for classifying images coming from other pairs of scanners. The average classification accuracy between scanners of the exact same model in Table 11 is less than other pairs, possibly because both these scanners have the same mechanical structure and use exactly the same post-processing algorithms. When compared to similar scenarios in Table 2 and Table 4, it is very clear that statistical feature vector based SVM classification performs much better than correlation based approaches.

Tables 12 and 13 show the confusion matrices for classifying three and four scanners respectively. An average classification accuracy of approximately $96 \%$ shows the effectiveness of our approach for source scanner identification. 


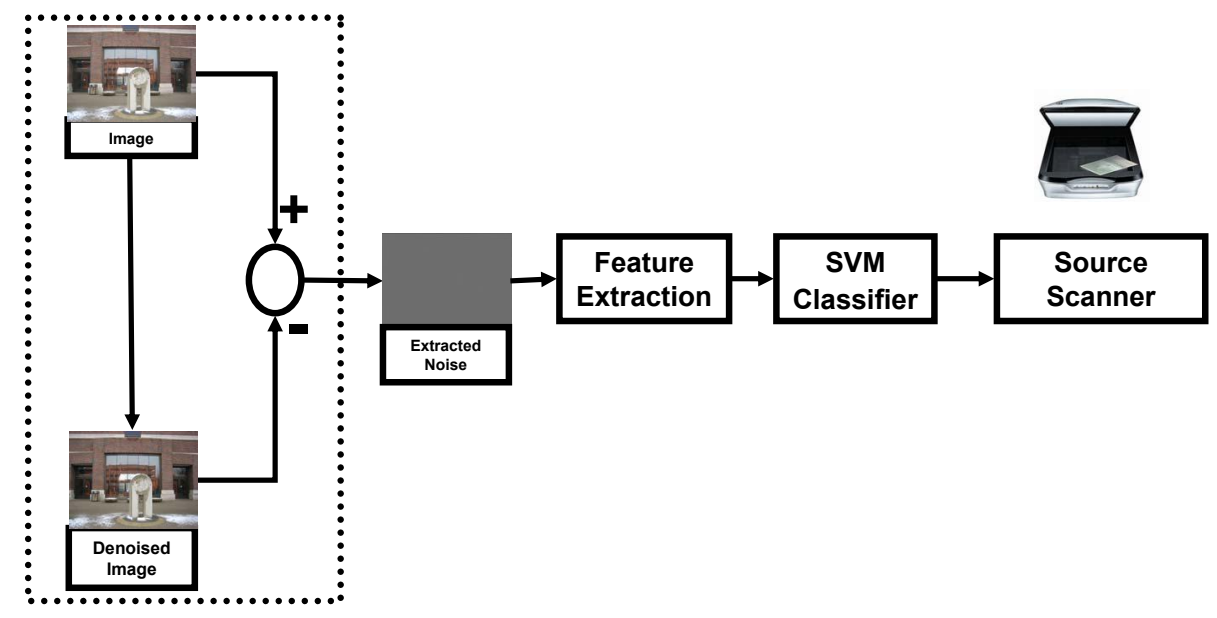

Figure 6. Scanner identification

Table 8. Confusion Matrix for Experiment 3 (SVM)

\begin{tabular}{|c|c|c|c|}
\hline \multicolumn{2}{|c|}{} & \multicolumn{2}{c|}{ Predicted } \\
\cline { 3 - 4 } \multicolumn{2}{c|}{} & $S_{1}^{1}$ & $S_{1}^{2}$ \\
\hline \hline \multirow{2}{*}{ Actual } & $S_{1}^{1}$ & 59.1 & 40.9 \\
\cline { 2 - 4 } & $S_{1}^{2}$ & 24.5 & 75.5 \\
\hline
\end{tabular}

Table 9. Confusion Matrix for Experiment 3 (SVM)

\begin{tabular}{|c|c|c|c|}
\hline \multicolumn{2}{|c|}{} & \multicolumn{2}{c|}{ Predicted } \\
\cline { 3 - 4 } \multicolumn{2}{|c|}{} & $S_{1}$ & $S_{2}$ \\
\hline \hline \multirow{2}{*}{ Actual } & $S_{1}$ & 100.0 & 0.0 \\
\cline { 2 - 4 } & $S_{2}$ & 0.9 & 99.1 \\
\hline
\end{tabular}

Figure 7 shows the effect of changing the size of the training dataset on average classification accuracy when classifying among four scanners. The total number of images used is approximately 1200.

To further examine the robustness of our approach, we tested it on images that have been JPEG compressed. We stored all the scanned sub-images in JPEG format and trained the SVM classifier on 50\% of the compressed images and then tested on the rest of the compressed images. An average classification accuracy of $85 \%$ is obtained for images with JPEG quality factor 90, as indicated by the confusion matrix shown in Table 14 .

All the above experiments were done with images scanned at the native resolution of the scanners. Further experiments need to be performed to check the efficacy of the method for images that have undergone further post-processing such as sharpening and blurring. Also, experiments need to be performed to check the accuracy of the method on classifying images scanned below the native resolution of the scanner. This last experiment has a broad practical impact as most images scanned by common users are at low resolutions due to limitations on storage space and difficulty of transmission.

\section{CONCLUSION AND FUTURE WORK}

In this paper we investigated the use of imaging sensor pattern noise for source scanner identification. The results in Tables 6, 7 and 12 show that the statistical feature vector based method performs much better than

Table 10. Confusion Matrix for Experiment 3 (SVM)

\begin{tabular}{|c|c|c|c|}
\hline \multicolumn{2}{|c|}{} & \multicolumn{2}{c|}{ Predicted } \\
\cline { 3 - 4 } \multicolumn{2}{|c|}{} & $S_{1}$ & $S_{4}$ \\
\hline \hline \multirow{2}{*}{ Actual } & $S_{1}$ & 100.0 & 0.0 \\
\cline { 2 - 4 } & $S_{4}$ & 0.0 & 100.0 \\
\hline
\end{tabular}

Table 11. Confusion Matrix for Experiment 3 (SVM)

\begin{tabular}{|c|c|c|c|}
\hline \multicolumn{2}{|c|}{} & \multicolumn{2}{c|}{ Predicted } \\
\cline { 3 - 4 } \multicolumn{2}{|c|}{} & $S_{2}$ & $S_{3}$ \\
\hline \hline \multirow{2}{*}{ Actual } & $S_{2}$ & 89.8 & 10.2 \\
\cline { 2 - 4 } & $S_{3}$ & 6.3 & 93.7 \\
\hline
\end{tabular}


Table 12. 3 Scanner Confusion Matrix for Experiment 3 (SVM)

\begin{tabular}{|c|c|c|c|c|}
\hline \multicolumn{2}{|c|}{} & \multicolumn{3}{|c|}{ Predicted } \\
\cline { 3 - 5 } \multicolumn{2}{|c|}{} & $S_{1}$ & $S_{2}$ & $S_{4}$ \\
\hline \hline \multirow{3}{*}{ Actual } & $S_{1}$ & 100.0 & 0.0 & 0.0 \\
\cline { 2 - 5 } & $S_{2}$ & 0.4 & 98.6 & 1.0 \\
\cline { 2 - 5 } & $S_{4}$ & 0.0 & 2.8 & 97.1 \\
\hline
\end{tabular}

Table 13. 4 Scanner Confusion Matrix for Experiment 3 (SVM)

\begin{tabular}{|c|c|c|c|c|c|}
\hline \multicolumn{2}{|c|}{} & \multicolumn{4}{|c|}{ Predicted } \\
\cline { 3 - 6 } \multicolumn{2}{c|}{} & $S_{1}$ & $S_{2}$ & $S_{3}$ & $S_{4}$ \\
\hline \hline \multirow{3}{*}{ Actual } & $S_{1}$ & 100.0 & 0.0 & 0.0 & 0.0 \\
\cline { 2 - 6 } & $S_{2}$ & 0.0 & 90.5 & 8.5 & 1.0 \\
\cline { 2 - 6 } & $S_{3}$ & 0.7 & 3.1 & 95.3 & 1.0 \\
\cline { 2 - 6 } & $S_{4}$ & 0.0 & 1.1 & 1.4 & 97.5 \\
\hline
\end{tabular}

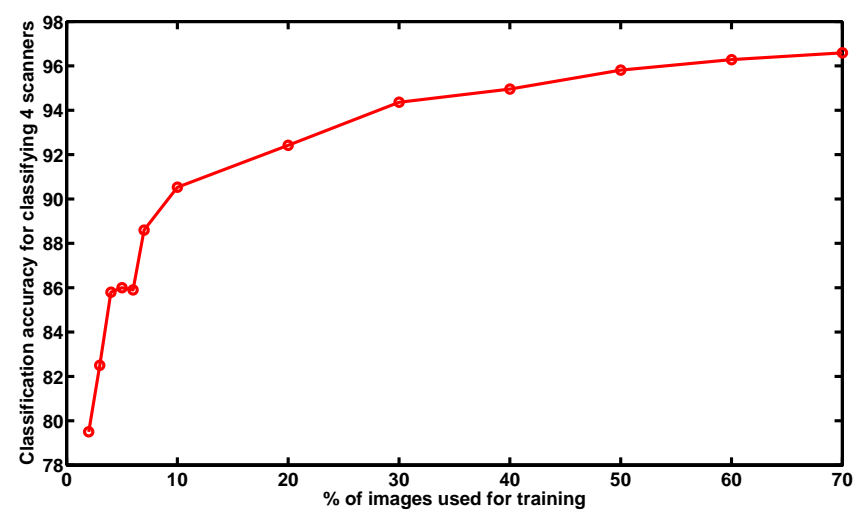

Figure 7. Effect of Training Dataset Size on Average Classification Accuracy.

the correlation based approaches used for source camera identification. Selection of proper features is the key to achieve accurate results. For classifying four scanners (two of which are the exact same model), an average classification accuracy of $96 \%$ is obtained. Table 14 shows that the proposed scheme performs well even with images that have undergone JPEG compression. It will also be important to extend this technique to work with images scanned at resolutions other than the native resolutions of the scanners. The challenge in working with lower resolution is to somehow address the degradation in the sensor noise pattern due to down sampli! ng. Future work will also include tests on images that have undergone various filtering operations such as sharpening, contrast stretching, and resampling. We are also looking at extending this approach for forgery detection.

\section{REFERENCES}

1. S. O. Jackson and J. Fuex. (2002) Admissibility of digitally scanned images. [Online]. Available: www.iediscovery.com/news/AdmissibilityDigitalImages.pdf

2. N. Khanna, A. K. Mikkilineni, A. F. Martone, G. N. Ali, G. T.-C. Chiu, J. P. Allebach, and E. J. Delp, "A survey of forensic characterization methods for physical devices," Digital Investigation, vol. 3, pp. 17-28, 2006.

3. M. Kharrazi, H. T. Sencar, and N. D. Memon, "Blind source camera identification," Proceedings of the IEEE International Conference on Image Processing, 2004, pp. 709-712.

4. A. Popescu and H. Farid, "Exposing digital forgeries in color filter array interpolated images," IEEE Transactions on Signal Processing, vol. 53, no. 10, pp. 3948-3959, 2005.

5. S. Bayram, H. Sencar, N. Memon, and I. Avcibas, "Source camera identification based on cfa interpolation," Proceedins of the IEEE International Conference on Image Processing, 2005, pp. 69-72.

6. J. Lukas, J. Fridrich, and M. Goljan, "Determining digital image origin using sensor imperfections," Proceedings of the SPIE International Conference on Image and Video Communications and Processing, A. Said and J. G. Apostolopoulos, Eds., vol. 5685, no. 1. SPIE, 2005, pp. 249-260. 
Table 14. Confusion Matrix for JPEG Images Using SVM

\begin{tabular}{|c|c|c|c|c|c|}
\hline \multicolumn{2}{|c|}{} & \multicolumn{4}{|c|}{ Predicted } \\
\cline { 3 - 6 } \multicolumn{2}{|c|}{} & $S_{1}$ & $S_{2}$ & $S_{3}$ & $S_{4}$ \\
\hline \hline \multirow{4}{*}{ Actual } & $S_{1}$ & 98.4 & 0.5 & 1.0 & 0.0 \\
\cline { 2 - 6 } & $S_{2}$ & 0.0 & 68.3 & 29.8 & 1.9 \\
\cline { 2 - 6 } & $S_{3}$ & 3.40 & 14.3 & 78.2 & 4.1 \\
\cline { 2 - 6 } & $S_{4}$ & 1.8 & 2.3 & 0.6 & 95.3 \\
\hline
\end{tabular}

7. N. Khanna, A. K. Mikkilineni, G. T.-C. Chiu, J. P. Allebach, and E. J. Delp, "Forensic classification of imaging sensor types," Proceedings of the SPIE International Conference on Security, Steganography, and Watermarking of Multimedia Contents IX, 2007, to appear in.

8. Z. J. Geradts, J. Bijhold, M. Kieft, K. Kurosawa, K. Kuroki, and N. Saitoh, "Methods for identification of images acquired with digital cameras," Enabling Technologies for Law Enforcement and Security, S. K. Bramble, E. M. Carapezza, and L. I. Rudin, Eds., vol. 4232, no. 1. SPIE Press, 2001, pp. 505-512.

9. G. C. Holst, CCD Arrays, Cameras, and Displays, Second Edition. JCD Publishing \& SPIE Press, USA, 1998.

10. M. K. Mihcak, I. Kozintsev, K. Ramchandran, and P. Moulin, "Low-complexity image denoising based on statistical modeling of wavelet coefficients," IEEE Signal Processing Letters, vol. 6, no. 12, pp. 300-303, 1999.

11. J. Tyson. (2001) How scanners work. [Online]. Available: http://computer.howstuffworks.com/scanner.htm

12. (2001, Nov.) Scanners. [Online]. Available: http://www.pctechguide.com/55Scanners.htm

13. J. R. Janesick, Scientific Charge-Coupled Devices. SPIE, Jan 2001.

14. J. Lukas, J. Fridrich, and M. Goljan, "Detecting digital image forgeries using sensor pattern noise," Proceedings of the SPIE International Conference on Security, Steganography, and Watermarking of Multimedia Contents VIII, vol. 6072, San Jose, CA, January 2006.

15. A. Foi, V. Katkovnik, K. Egiazarian, and J. Astola, "A novel local polynomial estimator based on directional multiscale optimizations," Proceedings of the 6th IMA Int. Conf. Math. in Signal Processing, vol. 5685, no. 1, 2004, pp. 79-82.

16. C. J. C. Burges, "A tutorial on support vector machines for pattern recognition," Data Mining and Knowledge Discovery, vol. 2, no. 2, pp. 121-167, 1998.

17. N. Cristianini and J. Shawe-Taylor, An introduction to support vector machines (and other kernel-based learning methods). Cambridge University Press, 2000.

18. T. Joachims, "Making large-scale support vector machine learning practical," Advances in Kernel Methods: Support Vector Machines, B. Schölkopf, C. Burges, and A. Smola, Eds. MIT Press, Cambridge, MA, 1998. 